\author{
Military Technical College, \\ Kobry El-Kobbah, \\ Cairo, Egypt
}

$9^{\text {th }}$ International Conference

On Aerospace Sciences \&

Aviation Technology

\title{
MECHANISM OF ORGANIC REACTIONS UNDER THE EFFECT OF RADIOLYSIS
}

A Review Article

EL-DESSOUKY' M. M.

\section{ABSTRACT}

The mechanism of interaction of radiation especially with organic material explains the ways of optimum utilization of nuclear energy and to avoid its harmful effects. Nowadays three principal mechanisms are adopted to interpret the formation of radiolytic products. These three principal mechanisms are: free radical mechanism, excited state mechanism and interaction with solvated electrons. The presence of atmospheric oxygen, impurities, scavengers, as well as type of solvent, type of radiation, dose and dose rate affect on the mechanism of the radiolytic reaction. The adopted mechanisms are supposed according to the experimental found free radicals and radiolytic products.

The review collected most adopted mechanisms for the most important organic compounds. Summarized mechanisms for radiolysis of hydrocarbons, alkyl halides, olefins, unsaturated and aromatic hydrocarbons, alcohols, organic acids are shown. Also the mechanism of radiolysis of aqueous solutions of some organic compounds is offered. Finally some important technological applications of radiation á! @ offered.

\section{KEY WORDS}

Radiolysis, Mechanism of Radiolysis, Radiolysis of Organic Materials, Polymers, Dyes, Detoxification by Radiolysis, Treatment of Chlorinated Organic Compouds.

\footnotetext{
* Egyptian Armed Forces
} 


\section{1- INTRODUTION}

The fundamental studies in radiation chemistry aim to identify the various formed species during radiolysis, to understand the mechanism of their formation, to get the effect of absorbed dose and dose rate and to try to determine the intermediate and final stable end products [1]

The use of radiation, especially $\gamma$ and beta to initiate chemical reactions, is important in polymerization, controlled degradation, cross-linking and graftpolymerization. Much of information and results have been gathered on an empirical basis and development of fundamental knowledge that can be used to expect the improvements in materials via the use of radiation energy [2].

The biological effects of radiation are very important for treatment, bacterial and pharmaceutical sterilization, sterilization of seeds and preservation of food $[3,4]$.

\section{2- PRINCIPAL MECHANISMS OF GENERATION OF RADICALS DURING RADIOLYSIS OF ORGANIC COOMPOUNDS}

The three principal radiolytic mechanisms to interpret the production of free radicals as well as radiolytic products are $[1,5]$ :

Molecular excited mechanism, in which the excited molecules interact either with themselves or with other molecules to produce radicals and radiolytic products.

Free radical mechanism, in which the free radicals (formed due to deexcitation of the excited molecules) interact with solute, with the solvent molecules or with each others, or degradation occurs to produce radiolytic products.

Solvated electron mechanism?, in which solvated electron interact with reducible centers such as carbonyl gro'ups to reduce them to alcoholic groups or to react with positive molecules to produce excited ones, or to reduce higher oxidation state metal ion to lower one.

The three mechanisms participate simultaneously to produce radicals and radiolytic products and the predominiant one depends on:

- The nature of solvent

- The nature of solute.

- Presence or absence of oxygen.

- Absorbed dose and dose rate.

- Temperature and $\mathrm{pH}$ of solutions.

The nature of solvent depends on whether it is polar or not. Polar solvents facilitate the rapid formation and stabilization of solvated electrons that enable them to be more efficient for reduction such as in isopropyl alcohol.

The nature of solute affects on the direction of radiolytic mechanisms. If solute scavenge electrons, other mechanisms will be efficient for affecting on the solvent 
and the scavenging solute will be reduced. If the solute $S$ is keton, electrons can be scavenged, and free radicals may be scavenged also to produce radiolytic products [6].

Oxygen has an important role with ${ }^{\circ} \mathrm{H}$ to form ${ }^{\circ} \mathrm{O}_{2} \mathrm{H}$ and this changes the nature of radiolytic reactions to produce different radiolytic products. The nature of ${ }^{\circ} \mathrm{H}$ is reduction whereas that of ${ }^{\circ} \mathrm{O}_{2} \mathrm{H}$ is oxidation. The present solvated oxygen can scavenge easily the solvated electrons to form $\mathrm{O}_{2}^{-}$which reacts with $\mathrm{H}^{+}$to form $\cdot \mathrm{O}_{2} \mathrm{H}$ which has higher oxidizing tendency instead of reducing by solvated electrons in case of absence of oxygen. As a result, different radiolytic products may be formed due to different actions of formed species in absence and presence of atmospheric oxygen [7].

The dose rate and absorbed dose affect on the radiation yields due to the rapidity of some reactions than others. For examples, the physical and physicochemical changes are more effective at high dose rates due to their short period of reaction, whereas the chemical stage reactions are effective at moderate and low dose rates. The variation of absorbed dose and dose rate interprets the difference of Gvalues obtained during radiolysis of the same medium [6].

The difference in the working conditions of $\mathrm{pH}$ and temperature affects on the radiolytic mechanisms and reactions. For example, at lower $\mathrm{pH}$ value, the $\mathrm{H}^{+}$ acts as scavenger for electron to produce ${ }^{*} \mathrm{H}$ or facilitate the ability of oxygen molecule to scavenge electrons to produce $\mathrm{H}_{2} \mathrm{O}_{2}$. The temperature has an important effect on the rate of radiolytic reactions like normal reactions. The raise of temperature increases the radiolytic yield due to raise of energy levels of solute and solvent, and as a result less energy will be required for production of ion pairs and other excited species $[6,7]$.

The homolysis of excited molecules forms free radicals that are very reactive. They have unpaired electron and enter in many reactions in irradiated medium. The free radicals may react with stable molecule $M$ or another radical or ion forming intermediate or final products.

Sometimes, scavengers are added to react with some certain primary radical species. Some scavengers are specific for certain species (such as electrons). This can be utilized to measure the quantitative formation of these species. Other scavengers are less specific. At concentrations lower than $10^{-4} \mathrm{M}$, scavengers will react only with some diffusing species. Scavengers of concentrations of more than $10^{-4} \mathrm{M}$ will react with all diffusing species formed. High concentrations (over $0.1 \mathrm{M}$ ) are used to scavenge the primary species and inhibit the intermediate reactions. Sometimes, certain scavengers are added to remove certain primary species to prevent some reactions and to let another to proceed. Sometimes in the excited molecules, the energy is absorbed at certain location, and the effect appears at another susceptible site $[1,6]$. 


\section{1- The Hydrogen Atoms}

They are important reducing agents liberated during radiolysis of most solutions or hydrogen containing substances. They can liberate hydrogen molecules and radicals from organic compounds. Hydrogen radicals can be produced as a result of interaction of solvated electrons with $\mathrm{H}^{+}$in acidic solutions or directly from molecular species. In acidic solution, the hydrogen atoms predominate than solvated electrons. In existence of oxygen, they interact with it giving hydroperroxyl radical ${ }^{\circ} \mathrm{O}_{2} \mathrm{H}$ and become high effective oxidizing agent [7].

The main reactions of hydrogen radicals with organic compounds are addition (on double bond or in a ring) or hydrogen abstraction from- $\mathrm{CH}_{2}$ - groups of $\alpha$ positions to $-\mathrm{COOH},-\mathrm{OH}$ and withdrawing groups. They also produce with carbonyl compounds the corresponding alcohols and pinacols as follows $[6,7]$ :

$$
\begin{array}{lll}
\mathrm{H} \cdot & +\mathrm{RH} \longrightarrow \mathrm{R}+\mathrm{H}_{2} \\
\mathrm{PhH} & +\mathrm{H} \longrightarrow \mathrm{HPh} \cdot \mathrm{H} \\
\mathrm{RCH}_{2} \mathrm{OH}+\mathrm{H} \longrightarrow \mathrm{R} \dot{\mathrm{C}} \mathrm{HOH}+\mathrm{H}_{2}
\end{array}
$$

$\mathrm{Cl}$

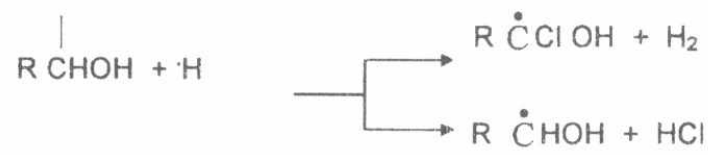

They also produce with carbonyl compounds the corresponding aicohols and pinacols

$$
\begin{aligned}
& \mathrm{H}+\mathrm{R}-\mathrm{C}-\mathrm{R} \longrightarrow \mathrm{R}-\mathrm{C} \cdot \mathrm{R}^{\mathrm{OH}}
\end{aligned}
$$

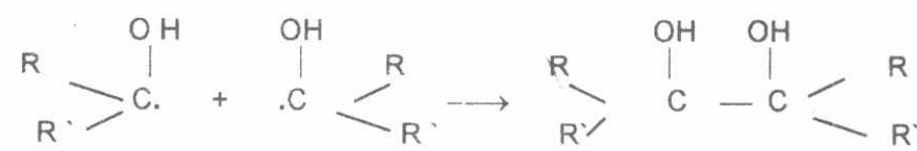

$$
\begin{aligned}
& \stackrel{\mathrm{R}}{\mathrm{R}}>\dot{\mathrm{C}}-\mathrm{OH}+\cdot \mathrm{H} \longrightarrow \stackrel{\mathrm{R}}{\longrightarrow} \mathrm{CH}-\mathrm{OH}
\end{aligned}
$$




\section{2- The Hydroxyl Radicals}

They are important oxidizing species that are formed during radiolysis of water and alcohols. In the reaction medium, they may show charge transfer (with ${ }^{-} \mathrm{OH}, \mathrm{X}^{-}$), hydrogen abstraction, addition or displacement as follows $[1,6]$ :

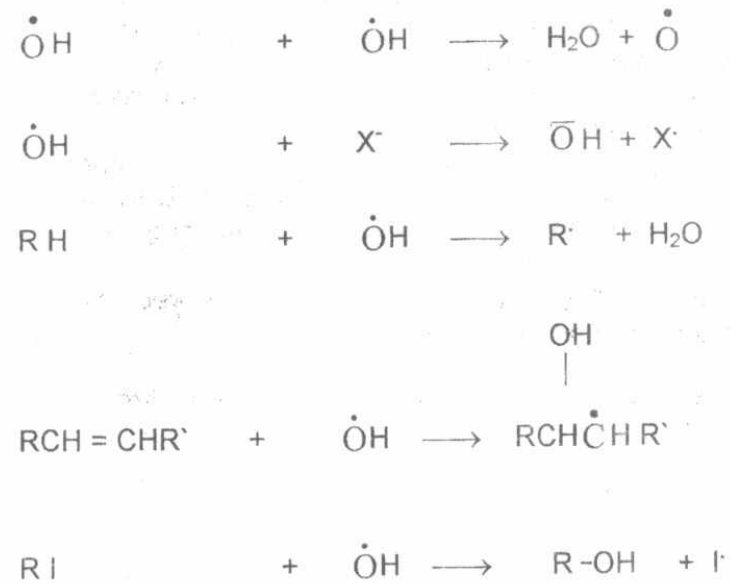

\section{3- Hydroperoxyl Radicals}

They have been identified as primary species in the tracks of heavy particles in aerated solution as a result of attack of $\mathrm{H}$ to $\mathrm{O}_{2}$ to get ${ }^{\circ} \mathrm{O}_{2} \mathrm{H}$ or as a result of interaction of negatively ionized oxygen molecule (as a result of interaction of $\mathrm{O}_{2}$ with electrons) with $\mathrm{H}^{+}$. They are considered as high oxidizing species [6].

Hydroperroxyl radicals have been identified as primary species in the tracks of heavy particles in deareated solution due to the following reaction :

$$
\dot{\mathrm{O}} \mathrm{H}+\mathrm{H}_{2} \mathrm{O}_{2} \longrightarrow \mathrm{H}_{2} \mathrm{O}+\mathrm{HO}_{2}^{\circ}
$$

In aerated solution, they are formed also as a result of attack of $\mathrm{H}^{\cdot}$ to $\mathrm{O}_{2}$

$$
\mathrm{H}+\mathrm{O}_{2} \longrightarrow \mathrm{HO}_{2}^{\circ}
$$

or as a result of interaction of negatively ionized oxygen molecule (as a result of interaction of $\mathrm{O}_{2}$ with electrons) with $\mathrm{H}^{+}$

$$
\overline{2} \dot{\mathrm{O}}+\mathrm{H}^{+} \longrightarrow \mathrm{HO}_{2}^{\circ}
$$




\section{4- Solvated Electrons}

They are the very active species that formed during radiolysis. They react as nucleophilic and their reductive property is very active and important. The main properties of free solvated electrons can be summarize:d as follows $[6,8,9]$ :

- If the solvent is pure water, the free solvated electrons have a broad optical absorption with maximum absorption at $720 \mathrm{m \mu}(\mathrm{nm})$. According to the type of solvent, they have absorption spectrum in UV, visible or IR.

- Solvated electrons react with oxidizing agents like sulfur, halogen, carbonyl (like ketones and aldehydes or nitro compounds).

- Solvated electrons have a period of life $>10^{-6} \mathrm{~s}$. They disappear according to the kinetic law of homogeneous; ion system. Their mobility is lower than those of electrons of metal conductors and higher than negative ions.

- They have magnetic moment. Therefore they can be followed by Electronic Paramagnetic Resonance (EPR)

The solvated electrons can be converted to stable anion using tetra nitro methane as scavenger as follows [10]:

$$
e_{h}^{-}+C\left(N_{2}\right)_{4} \rightarrow C\left(N_{2}\right)_{3}^{-}+N_{2}
$$

\section{5- Solute Species}

According to the type of solute, scavenging will be for certain radicals, electrons or excited species to produce solute species. Solute species may interact with solvent molecules or radicals to produce radiolytic final products. Some certain substances are more reactive towards radiation than others due to weakness of some bonds (like $\mathrm{S}-\mathrm{S}$ ), or due to high scavenging possibility of formed free radicals and electrons like urea, amines, and arnino acids. These substances can be utilized to prifect some biological systems against radiation damage or to determine quantitatively the formed radicals in order to utilize them for determination of absorbed rose $[3,6,8,9]$.

$$
\begin{array}{llll}
\mathrm{S}+ & \mathrm{e}_{\mathrm{S}}^{-} & \longrightarrow & \mathrm{S}^{\cdot} \\
\mathrm{S}+ & \mathrm{H}^{\cdot} & \longrightarrow & \mathrm{SH} \\
\mathrm{S}+ & \dot{\mathrm{C}} \mathrm{H} & \longrightarrow & \mathrm{SOH} \\
\mathrm{S}+ & \mathrm{M} & \longrightarrow & \mathrm{S}^{\cdot}+\mathrm{M} \\
& -\frac{d[S]}{d t}= & -\frac{d\left[R^{\bullet}\right]}{d t}=\frac{d[P]}{d t}=k_{i}[S]\left[R^{\bullet}\right]
\end{array}
$$

\section{3- RADIOLYSIS OF ORGANIC COMPOUNDS}

\section{1- Radiolysis of Hydrocarbons}

The mechanisms of radiolytic decomposition of organic compounds are not easy since the radiolytic products are too much to be analyzed. It can be expected that bonds of lower bond energy are broken before that of higher energy ones. 
Radiolysis of hydrocarbons showed that $\mathrm{C}-\mathrm{H}$ bond is broken easily than $\mathrm{C}-\mathrm{C}$. Radiolysis of organic compounds leads to formation of excited molecules, free radicals and ions that go through several reactions in the radiolytic medium. The excited molecules may induce reactions that give several radiolytic products, intermediate radicals or transfer energy to another molecules [6]. A simple mechanism can be represented as follows [10]:

$$
\begin{aligned}
& \text { initiation : } \\
& R H \stackrel{\gamma}{\longrightarrow} R^{\bullet}+H^{\bullet} \\
& \text { propagation: } \\
& R^{\bullet}+R H \rightarrow R-R+H^{\bullet} \\
& H^{\bullet}+R H \rightarrow R^{\bullet}+H_{2} \\
& \text { ter min ation : } \\
& R^{\bullet}+{ }^{\bullet} H \rightarrow R H \\
& R^{\bullet}+{ }^{\bullet} R \rightarrow R-R \\
& H^{\bullet}+{ }^{\bullet} H \rightarrow H_{2}
\end{aligned}
$$

Another mechanisms during radiolysis of organic compounds exist like proton transfer, radical generation from excited molecules, hydrogen abstraction and fragmentation of branched alkanes. Alkyl and hydrogen radicals that produced from excited species cause further degradation. Steric effects decrease the rate of recombination.

In aqueous solutions, degradation is formed by hydroxyl radical at $\mathrm{pH}$ greater than 6 and by hydrogen radicals at lower $\mathrm{pH}[11,12]$. The main effects of radiation on alkanes are: liberation of $\mathrm{H}_{2}$, development of unsaturation, formation of dimers, production of fragments from $C_{1}$ to $C_{n}$ and their combinations. Radicals will undergo dimerization, abstraction, and addition reactions. Electrons can be scavenged by nitrous oxide, sulfur hexafluoride, $\mathrm{CO}_{2}$, or aromatic compounds. Also water or ammonia can scavenge protons. Positive ions and free radical are scavenged by nitric acid. Free radials can be scavenged by iodine, $\mathrm{O}_{2}$, DPPH [ 1,1 diphenyl 2 pieryl hydrazyl]. Unsaturated produced positive ions and electrons can be scavenged by biphenyl and alkyl iodides. The presence and type of scavengers affect on the radiation yield. Mechanisms of ion molecules and ion molecule radicals interpret the formation of many radiolytic products $[13,14]$.

Ter-butanol acts as $\mathrm{OH}$ scavenger in aqueolıs oxygenated system forming terbutanol peroxy radical [15]. The haloginated hydrocarbons scavenge electrons and the produced $H X$ scavenge electrons too $[16,17]$. Baicalin scavenge hydroxyethyl and peroxyl radicals during radiolysis of aeratied ethanol in presence of oxygen. Baicalin molecule could inhibit formation of one molecule of hydrogen peroxide and two molecules of acetaldehyde during radiolysis of ethanol [18]. Hydroxy-cinnamic 
acid derivatives scavenge superoxide radical anion during radiolysis of organic acids [19].

\section{2- Alkyl Halides}

Usually their gas radiolytic products contain both halogens, $\mathrm{HX}$ and dimer as in case of dichloromethane [20]. Mechanism of reaction follows through excited state and free radical mechanisms. In aromatic halides, excess energy can be taken by the pi-electrons of benzene ring [21]. In radiolysis of chlorofluorocarbons, dechlorination was reported due to the effect of hydroxyl radjcals [22]. Modern utilization of radiolysis of halogen compounds is to get red of them as pollutants in industrial wastewater. Radiolysis with photolysis and using titanium oxide as catalyst proved good way for their detoxification specially that act as toxins and very difficult to be removed by classical chemical redox reactions. New attempts are directed to increase the radiation yield in their chain reactions [23]. More attempts are now made to utilize the spent reactor fuel as gamma radiation sources to decompose the halogenated hydrocarbons from wastewater [24].

Oxidative degradation of chlorinated organic compounds was proved due to surface adsorbed hydroxyl radicals on titanium oxide [25]. The presence of oxygen accelerates the chain reaction degradation and increases the effect of hydroxyl radical [26]. The main mechanisms are hole transfer for chlorinated radicals to cause electron transfer from the molecules as well as through free radical mechanisms $[27,28]$. The use of alcohol helps in degradation since hydroxyl radicals are formed radiolytically from them [29].

Electron attachment mechanism in halomethanes was proved [30]. In radiolysis of $\mathrm{CH}_{3} \mathrm{Br}$ and $\mathrm{CH}_{3} \mathrm{CH}_{2} \mathrm{Br}$ (which can be represented by $\mathrm{R}-\mathrm{Br}$ ) as elecron scavengers in tetrahydrofuran (HS). The yields of $\mathrm{CH}_{4}$ and $\mathrm{C}_{2} \mathrm{H}_{6}$ were formed due to this mechanism:

$$
\begin{aligned}
& e^{-}+\mathrm{RBr} \rightarrow \mathrm{R}^{\cdot}+\mathrm{Br}^{-} \\
& \mathrm{R}^{\cdot}+\mathrm{HS} \rightarrow \mathrm{RH}+\mathrm{S}^{-}
\end{aligned}
$$

Let $G(P)$ is the radiation yield of $\mathrm{CH}_{4}$ or $\mathrm{C}_{2} \mathrm{H}_{6}, \mathrm{C}$ is the concentration of $\mathrm{CH}_{3} \mathrm{Br}$ or $\mathrm{CH}_{3} \mathrm{CH}_{2} \mathrm{Br}, \mathrm{G}_{\text {esc }}$ is he yield of scaping spurs recombination, $\mathrm{G}_{\mathrm{gi}}$ is the yield of geminated recombination and $\alpha$ is adjustable parameter depending on the efficiency of scaping, then:

$$
G(P)=G_{e s c}+G_{g i} \frac{(\alpha C)^{0.5}}{1+(\alpha C)^{0.5}}
$$

For low concentration of $\mathrm{R}-\mathrm{Br}$ :

$$
G(P)=G_{e s c}+G_{g i}(\alpha C)^{0.5}
$$


For low concentration of $\mathrm{CH}_{3} \mathrm{Br}\left(1.51 \times 10^{-4}\right.$ to $\left.0.13 \mathrm{~mol}^{-1} \mathrm{l}^{-1}\right) \mathrm{G}_{\mathrm{esc}}=0.338 \pm 0.17$. $\mathrm{G}_{\mathrm{gi}}=3.82 \pm 0.24$ and $\alpha=19.8 \pm 3.4$ [31].

\section{3- Unsaturated Hydrocarbons and Cyclic Compounds}

They possess $\Pi$ 's orbital which differs significantly from $\sigma$-one of saturated hydrocarbons in having lower ionization potentials. The $\Pi$ 's orbital can add free radicals and ions. Presence of double bonds in molecule stabilizes the radical via resonance. Irradiation of liquid alkenes produces free ions of about the same yield as saturated hydrocarbons due to similar dielectric constants. The radiolysis of unsaturated hydrocarbons give products of multi-carbon atoms due to the ability of free radicals or cations to add to double bonds [12]. The main radicals detected during radiolysis of liquid alkenes are those from loss of a hydrogen atom from allyl positions and those corresponding to addition of hydrogen atoms at the double bond and the proposed mechanisms as follows [12] :

Polymer formation:

$$
\begin{aligned}
& \mathrm{C}_{n} \mathrm{H}_{2 n} \stackrel{\gamma}{\longrightarrow} \mathrm{C}_{n} \mathrm{H}_{2 n}^{+}+e^{-} \\
& \mathrm{C}_{n} \mathrm{H}_{2 n}^{+}+\mathrm{C}_{n} \mathrm{H}_{2 n} \rightarrow \mathrm{C}_{2 n} \mathrm{H}_{4 n}^{+} \\
& \mathrm{C}_{2 n} \mathrm{H}_{4 n}^{+}+e^{-} \quad \rightarrow \quad \mathrm{C}_{2 n} \mathrm{H}_{4 n}
\end{aligned}
$$

Cis-trans isomerization:

$$
\begin{aligned}
& R-\underset{H}{C}=\underset{H}{C}-R \quad-\quad H \quad \rightarrow \quad R-\dot{C}=\underset{H}{C}-R \\
& R-\dot{C}=\underset{H}{C}-R+{ }^{H} \rightarrow \stackrel{H}{C}=\underset{H}{C}-R
\end{aligned}
$$

Addition on the double bond:

$$
\begin{aligned}
& R-\underset{H}{C}=\underset{H}{C}-R+\cdot H \rightarrow R-\underset{H}{C}-\underset{H}{\stackrel{C}{C}}-R
\end{aligned}
$$

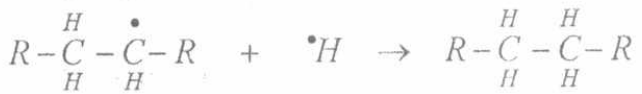

Formation of alkynes:

$$
\begin{aligned}
& R-\underset{H}{C}=\underset{H}{C}-\mathrm{R}+{ }^{\bullet} \mathrm{H} \rightarrow \mathrm{R}-\underset{\mathrm{C}}{\mathrm{C}}=\mathrm{C}_{\mathrm{H}}-\mathrm{R}+\mathrm{H}_{2} \\
& \mathrm{R}-\mathrm{C}=\mathrm{C}-\mathrm{R}+\mathrm{H}_{\mathrm{H}} \mathrm{H} \rightarrow \mathrm{R}-\mathrm{C} \equiv \mathrm{C}-\mathrm{R}+\cdot \mathrm{H}_{2}
\end{aligned}
$$


The radiolysis of cyclohexane may produce dimerization of cyclohexyl radicals. In substituted cyclohexane, the mechanism of production of of radiolytic products was through excitation energy transfer [32]. Hydrogen gas was obtained as principal product due to radiolysis of liquid cyclohexane [33]. During radiolysis of cyclohexane in presence of oxygen, hydroperoxide radical with cyclohexane ones are produced $[34,35]$.

In cyclohexane :

$$
G(P)=G_{e s c}\left[1+\left(1.61 r_{c}{ }^{2} k C / D\right)^{0.6}\right]
$$

Where $r_{c}$ is Onseger escape radius, $k$ is rate constant of scavenging of electron by solute, $C$ is concentration of scavenger and $D$ is the sum of diffusion coefficient of electron and counter ions.

Radiolysis of cyclopentane yields cyclopentene and bicyclopentyl radical [36]. Radiolysis of fluorohexane produces filuorine $[37,38]$.

\section{4-Aromatic Hydrocarbon}

Due to conjugated electrons, aro'matics resist irradiation. This resistivity is due to the ability of conjugated system to degrade excitation energy without localizing it on certain bond, and hence there is; no scission. Thiophenol is the only aromatic that is reported to be sensitive to radiation due to ability of $\mathrm{C}-\mathrm{S}$ bond to be ruptured [39] :

The aromatic ring scavenges; radicals, electrons, and $\mathrm{H}_{2}$. During their $\gamma$ irradiation, polymer, as well as ethers are produced. The presence of different scavengers at different doses affects the degradation yield and its mechanism. Alkyl substitution increases the sensitivity to radiation and abstraction is less than addition [39]. The radiolysi's of substituted benzenes in aqueous solution involves both direct electron transfe:r, addition and elimination reactions $[40,41,42]$.

Addition of oxygen on oriho and para positions to hydroxyl group in cresols during radiolysis was investiga'ced [43]. In radiolysis of vapor form with other compounds and water, substitution of groups takes piace by hydrogen atom, hydroxyl radicals or other radicals defsending on the nature of existing material and ratio of composition of vapor as follows [44]:

$$
\begin{aligned}
& \mathrm{H}^{\bullet} \text { or }{ }^{\bullet} \mathrm{OH}+\mathrm{C}_{6} \mathrm{H}_{6} \rightarrow{ }^{\bullet} \mathrm{C}_{6} \mathrm{H}_{5}+\mathrm{H}_{2} \text { or } \mathrm{H}_{2} \mathrm{O} \\
& { }^{\circ} \mathrm{C}_{6} \mathrm{H}_{5}+{ }^{\circ} \mathrm{OH} \rightarrow \mathrm{C}_{6} \mathrm{H}_{5}-\mathrm{OH}
\end{aligned}
$$

\section{5- Alcohols}

The properties of simple alcohols are intermediate between those of water, which contains only $(\mathrm{O}-\mathrm{H})$ bonds, and the saturated hydrocarbons, which contain only 
( $\mathrm{C}-\mathrm{H}$ ) bonds. The simple alcohols have dielectric constants at ordinary temperature of 20 - 30, compared with 80 for water and 2 for hydrocarbons. Therefore, the results obtained during their radiolysis are between those found from the two [45].

Aliphatic alcohols scavenge solvated electrons but not strongly as in water Isobutanol scavenge hydroxyl radicals to form aldehyde [44]. Radiolysis of isopropyl alcohol can be utilized as a source of producing hydroxyl radicals for detoxification of halogenated waste [45]. The mechanism of radiolysis of methano alikes that of glycerin. The principal mechanisms of interactions of solvated electrons and excited molecules in alcohols cooperate to form radiolytic products as follows $[47,47]$

$$
\begin{aligned}
& e_{\text {sol }}^{-}+\mathrm{R}-\mathrm{CH}_{2}-\mathrm{OH} \rightarrow \mathrm{R}-\mathrm{CH}_{2}-\mathrm{O}^{-}+\cdot \mathrm{H} \\
& 2^{\bullet} \mathrm{OH}+\mathrm{R}-\mathrm{CH}_{2}-\mathrm{OH} \rightarrow \mathrm{R}-\mathrm{COH}+2 \mathrm{H}_{2} \mathrm{O}
\end{aligned}
$$

The existence of scavengers lessens the yield of radiolytic products $[49,50]$.

\section{6- Radiolysis of Ketones}

The radiochemical yields of main products during $\mathrm{y}$-radiolysis of furyl ketones in 2-propanol were studied. The yields of hydrogen and methane were lower than those obtained from 2-propanol only. Concerning the mechanism, electron capture and radical processes are probably the reason of formation of the alcohol and pinacols which are produced by radioreduction and dimerization respectively. Substitution products indicated radical processes as follows[51]:

$$
\begin{aligned}
& H^{\bullet}+{ }_{R}^{R} \cdot C=O \rightarrow \stackrel{R}{R} \stackrel{\bullet}{C}-O H \\
& { }_{R}^{R} \cdot \dot{C}-\mathrm{OH}+H^{\bullet} \rightarrow \quad{ }_{R}^{R} \mathrm{CH}-\mathrm{OH} \\
& \begin{array}{l}
R \\
R
\end{array} \dot{C}-\mathrm{OH}+{ }_{R}^{R} \dot{C}-\mathrm{OH} \rightarrow \underset{R}{R} \stackrel{\mathrm{OH}}{\mathrm{CH}-\stackrel{O H}{\mathrm{CH}}} \stackrel{R}{R}
\end{aligned}
$$

\section{7- Radiolysis of Organic Acids}

The radiolysis of formic acid as an example is studied; since it is good scavenger for electrons hydrogen and hydroxyl radicals. The $G$ value depends on $\mathrm{pH}$, 
solution concentration, medium, dose and dose rate. Irradiation of carboxylic acids produce large quantities of $\mathrm{H}_{2}, \mathrm{CO}_{2}$ as follows:

$$
\begin{aligned}
\mathrm{HCOOH}+{ }^{\cdot} \mathrm{Hor}{ }^{\circ} \mathrm{OH} & \rightarrow{ }^{\circ} \mathrm{COOH}+\mathrm{H}_{2} \mathrm{or} \mathrm{H}_{2} \mathrm{O} \\
2{ }^{\circ} \mathrm{COOH} & \rightarrow \mathrm{COOH} \\
& \rightarrow \mathrm{COOH}_{2}+\mathrm{CO}_{2} \mathrm{HOOH}
\end{aligned}
$$

The gas phase during their radiolysis contains $\mathrm{CO}_{2}$ and light hydrocarbon. The formation of radiolytic products increases with temperature [52,53].

\section{8- Radiolysis of Ethers}

Ethers are attacked by hydroxyl radicals generated by radiation [54]. Most radiolytic mechanisms are based on experimental results, like rupture of $\mathrm{C}-\mathrm{O}$ bond [55] . Also free radical mechanism for formation of radiolytic products was adopted due to degradation of $\mathrm{C}-\mathrm{O}, \mathrm{C}-\mathrm{H}$ bonds. Beside radical interaction mechanism, both ionic and excitation mechanisms are op erative [56] .

\section{9- Radiolysis of Sorne Organic Aqueous Solutions}

Neutral aerated aqueous solution of ethanol gives acetaldehyde. In deaerated solutions, $\mathrm{CH}_{3} \dot{\mathrm{C}} \mathrm{HOH}$ is formed from the reaction of $H$ and $\dot{\mathrm{O}} \mathrm{H}$ with the molecule of alcohol. Two of the formed radicals give the corresponding pinacol. In acidic deaerated solution, acetaldehyde and pinacol are formed $[57,58]$. In neutral aerated aqueous solution of ethanol gives acetaldehyde as follows

$$
\begin{aligned}
& \mathrm{CH}_{3} \mathrm{CH}_{2} \mathrm{OH}+\dot{\mathrm{OH}} \longrightarrow \mathrm{CH}_{3} \dot{\mathrm{C}} \mathrm{H}-\mathrm{OH}+\mathrm{H}_{2} \mathrm{O} \\
& \mathrm{CH}_{3} \mathrm{CHOH}+\mathrm{O}_{2} \longrightarrow \mathrm{CH}_{3} \mathrm{CHO}+\mathrm{HO}_{2}
\end{aligned}
$$

In deaerated solutions $\mathrm{CH}_{3} \dot{\mathrm{C}} \mathrm{HOH}$ is formed from the reaction of $\mathrm{H}$ and $\dot{\mathrm{O}} \mathrm{H}$ with the molecule of alcohol. Two of the formed radicals give the corresponding glycol:

$$
\begin{array}{ccc}
\dot{\mathrm{O}}+ & 2 \mathrm{CH}_{3} \mathrm{CH}_{2} \mathrm{OH} \longrightarrow 2 \mathrm{CH}_{3} \dot{\mathrm{C}} \mathrm{HOH}+\mathrm{H}_{2}+\mathrm{H}_{2} \mathrm{O} \\
\mathrm{CH}_{3} \dot{\mathrm{C}} \mathrm{HOH}+\mathrm{CH}_{3} \dot{\mathrm{C}} \mathrm{HOH} \longrightarrow & \mathrm{CH}_{3} \mathrm{CH} \mathrm{OH} \\
\mathrm{CH}_{3} \mathrm{CH} \mathrm{OH}
\end{array}
$$


In radiolysis of $0.25 \mathrm{M}$ aqueous solution $\mathrm{t}-\mathrm{BuOH}, \mathrm{OH}$ is scavenged with $\mathrm{k}=6$ $\times 10^{8} \mathrm{M}^{-1} \mathrm{~s}^{-1}$, whereas $\mathrm{OH}$ and $\mathrm{H}$ species react with $0.17 \mathrm{M}$ t-PrOH with $\mathrm{k}=$ $1.9 \times 10^{9} \& 7.4 \times 10^{7} \mathrm{M}^{-1} \mathrm{~s}^{-1}$ respectively. In alkaline medium $e^{-}$is produced from reaction of $\cdot \mathrm{H}$ with $\mathrm{OH}\left(k=2.2 \times 10^{7} \mathrm{M}^{-1} \mathrm{~s}^{-1}\right)$, whereas in acidic medium $\mathrm{e}_{\text {aq }}^{-}$ reacts with $\mathrm{H}^{+}$to give $\mathrm{H}\left(\mathrm{k}=2.3 \times 10^{10} \mathrm{M}^{-1} \mathrm{~s}^{-1}\right)$ as follows:

At $\mathrm{pH} 13, \mathrm{G}$ of $\mathrm{i}-\mathrm{PrOH}$ was independent of dose and equals 3.2 and $\mathrm{G}$ of $\mathrm{t}-\mathrm{PuOH}$ equals $6.6[59]$.

3.10- Radiolysis of Chlorinated Oils

$$
\begin{aligned}
& \mathrm{H}_{2} \mathrm{O} \quad \rightarrow \quad e_{a q}^{-}(2.6)+{ }^{\bullet} \mathrm{OH}(2.7)+\mathrm{H}^{\cdot}(0.6) \\
& \mathrm{HO}^{\bullet}+\left(\mathrm{CH}_{3}\right)_{3} \mathrm{COH} \stackrel{6 \times 10^{8}}{\longrightarrow}{ }^{\bullet} \mathrm{CH}_{2}\left(\mathrm{CH}_{3}\right)_{2} \mathrm{COH}+\mathrm{H}_{2} \mathrm{O} \\
& \mathrm{HO}^{\bullet}+\left(\mathrm{CH}_{3}\right)_{2} \mathrm{CHOH} \stackrel{1.9 \times 10^{9}}{\longrightarrow}\left(\mathrm{CH}_{3}\right)_{2} \stackrel{\bullet}{\mathrm{C}}-\mathrm{OH}+\mathrm{H}_{2} \mathrm{O} \\
& \mathrm{H}^{\bullet}+\left(\mathrm{CH}_{3}\right)_{2} \mathrm{CHOH} \stackrel{7.4 \times 10^{7}}{\longrightarrow}\left(\mathrm{CH}_{3}\right)_{2} \dot{\mathrm{C}}-\mathrm{OH}+\mathrm{H}_{2} \mathrm{O} \\
& \mathrm{H}^{\bullet}+\mathrm{OH}^{-} \stackrel{2.7 \times 10^{7}}{\longrightarrow} \bar{e}_{a q}+\mathrm{H}_{2} \mathrm{O} \\
& e_{a q}^{-}+H^{+} \stackrel{2.3 \times 10^{10}}{\longrightarrow} H^{\bullet}
\end{aligned}
$$

Radiolysis of hydraulic and transformer oils was studied. The result of radiolysis was that dechlorination had occurred [60]. The same mechanism occurred in radiolysis of polychlorinated phenols in several oils. This dechlorination process by radiation can be utilized as an advanced way for dechlorination in waste treatment of toxic chlorinated compounds $[59,60]$.

\section{4- MECHANISM OF IRRADIATION OF POLYMER}

The mechanisms of irradiation of polymers are deduced from changes in structure and properties of irradiated polymer, or from the properties of prepared polymers by irradiation. The analysis of effects of radiation was studied by Electron Spin Resonance (ESR), Nuclear Magnetic Resonance (NMR), Gas Chromatography (GC). Fourier Transformed Infrared (FTIR), Differential Scanning Calorimetry (DSC), Thermogravimetric Analysis (TGA), and Dynamic Mechanical Analysis (DMA) [2]. In copolymerization by radiation, cationic and radical mechanisms were proved [61].

Both degradation during irradiation and graft polymerization in post irradiation occur through radical mechanism [62]. The dependance of cission as a function of LET was almost constant at low LET and decreased at high LET ion irradiation [63] . The scission chain reaction decreases with lowering temperature [64]. The scisssion does not take place immediately after absorption of radiation energy, but it is induced by the intramolecular radical conversion [65]. Some changes 
may be attributed to trapping of negative charge on certain electronegative groups or benzene rings [66]. The mechanical properties of polymers are deteriorated by radiation and additional crosslinking beside degradation have occurred. Thermal annealing may be useful for recovery of mechanical properties [67]. The mechanism of free radical polymerization can be considered in terms of four elementary steps as follows[68]:

1-Formation of free radicals $R$.

The rate of decomposition $R_{d}$ is:

$$
\begin{gathered}
I \stackrel{k_{d}}{\longrightarrow} R^{\bullet}+R^{\bullet} \\
\mathrm{R}_{\mathrm{d}}=\mathrm{k}_{\mathrm{d}}[\mathrm{I}]
\end{gathered}
$$

Where $k_{d}$ is radical formation rate constant $\left(I \cdot \mathrm{M}^{-1} \cdot \mathrm{s}^{-1}\right)$ and [I] is the concentration in ${\mathrm{M} . \mathrm{I}^{-1}}^{-1}$

2-Addition of the first monomer molecule $M$ with radical $R$ to initiate the chain reaction:

$$
\begin{aligned}
R^{\bullet}+M & \stackrel{k_{i}}{\longrightarrow} R M^{\bullet} \\
R_{i} & =k_{i}\left[R^{\bullet}\right][M]
\end{aligned}
$$

Where $k_{1}$ is chain initiation rate constant

3-Propagation reaction to add monomers with propagation rate constant $k_{p}$ and propagation rate $R_{p}$ as follows:

$$
\begin{gathered}
R M^{\bullet}+n M \stackrel{k_{p}}{\longrightarrow} R M n^{\bullet} \quad\left(\text { or } P^{\bullet}\right) \\
R_{p}=\frac{d\left[P^{\bullet}\right]}{d t}=k_{p}\left[R M^{\bullet}\right][n M]
\end{gathered}
$$

Where $[\mathrm{nM}]$ is the monomer concentration, $[\mathrm{P}]$ is the sum of concentration of all $\mathrm{RM}$

4-Bimolecular termination reaction which can be represented by the termination rate constant $k_{t}$ and rate of termination as follows:

$$
\begin{gathered}
R M_{n}^{\bullet}+R M_{n}^{\bullet} \stackrel{k_{t}}{\longrightarrow} \text { Unreactive Species }(Y) \\
R_{t}=-\frac{d[Y]}{d t}=k_{t}\left[P^{\bullet}\right]^{2}
\end{gathered}
$$




\section{REFERENCES}

[1] Nacasek -F., Nuclear Chemistry 2 Ed., Chap. 4, Prague Academia , p. 131-176 1992.

[2] Carswell-Pomerantz -T., Babanalbandi -A., Dong -L., Hill -D.J.T., Perera-M.C.S. Pomery-P.J., Saadat-G., Whittaker-A.K., International Atomic Energy Agency, Vienna (Austria) Project 1994-1997, 147 p., p. 111-128, Jan 1999.

[3] Lau H. M., Investigation of Damage Mechanism by lonizing Radiation on Biomolecules, COSTAM Kuala Lumpur (Malaysiaı), p.7, 1996

[4] Nawar -W.W., Zhu -Z., Wan -H., DeGroote -E., Chen -Y., Aciukewicz -T., Progress in the Detection of Irradiated Foods by Mleasurement of Lipid-Derived Volatiles, Intern. Meeting on Analytical Detection Methods for Irradiation Treatment of Foods. Belfast, Northern Ireland (United Kingdom). 20-24 Jun 1994., Proceedings. Cambridge (United Kingdom). Royal Society of Chemistry, p. $241-248,1996$

[5] El-Dessouky -M. M., Gar-Alam Rashed -1.," Radiolysis of 1-(2-Furanyl)-1Pentanone in the Presence of Atmospheric Oxygenn", J. of Radioanal. and Nucl. Chem., V. 92(1), p. 51-57, 1985

[6] El-Dessouky -M. M., Contribution of Study of the $\gamma$ Radiolysis of Furanyl Ketones in the 2-Propanol, Ph.D. Thesis, INP No.189, Toulouse (France), 1982.

[7] El-Dessouky -M. M., Gar Alam Rasshed -I.," A Study on the $\gamma$ Radiolysis of 2Furanyl Ketones in 2-Propanol,ir Presence and Absence of Atmospheric Oxygen", MEJ (Mansoura Eng. J.), V. 14(2), p. 1-9, 1989.

[3] Strauss -B. S., Factors Affecting Mutational Specifity Induced by lonizing Radiation and Oxidizing Radicals. Progress Report, Chicago Univ., USA, p. 15, 1992.

[9] Zhao -Yixing, Freemab -G. R..,"Solvated Electron Mobility in Liquid TerButanol", Canadian J. of Chem. , V. 73(3), p. 389-391, 1995.

[10] Mozumder, -A . Fundamentals of Radiation Chemistry, Acad. Press , London, p. 154-372, 1999

[11.] Shkrob -1. A., Trifunac -A. D.," Pulse Radiolysis of Alkanes: A Time-Resolved EPR.Study - Part I. Alkyl Radicals", Rad. Phys. and Chem. , V. 46(1), p. 8396, 1995.

[12] Maity -D. K., Heri -M.," Reaction of Hydroxyl Radicals with 1-Bromo-nlodoalkane in Aqueous Solutions", Trombay Symp. on Rad. and Photochemistry, Bombay (india), p. 28-30, 1996. 
[13] Feldman -V. L., Sukhov -F. F., Slovokhotova -N A.,"Radiation -Induced Degradation of Alkane Molecules in Solid Rare Gas Matriceses", Rad. Phys. and Chem. , V. 48(3), p. 261-269, 1996.

[14] Werst -D. W., Trifunac -A. D.," Early Events Following Radiolytic and Photogeneration of Radical Cations in Hydrocarbon" , Int. Conf. On Rad. Tolerant Scintillators and Detectors, Tallahassee, USA, $28 \mathrm{Apr}-2$ May, p. 10,1992

[15] Piechowski-M. -Von., "Ter-Butanol as an $\mathrm{OH}$-Scavenger in the Radiolysis of Oxygenated Aqueous Systems", Berichte-der-Bunsengesellschaft-fuerPhysikalische-Chemie, V. 96(10), p. 1448-1454, 1992.

[16] Suwalski -J. P., Kroh -J.," The Yields of Intermediates , $\mathrm{HCl}$ and Scavengeable Electrons in the Irradiated $\mathrm{MCH} /$ Iso-Propanol / $\mathrm{CCl}_{4}$ at 4,6 and $77 \mathrm{~K}^{\prime \prime}$, Bull. of the Polish Acad. of Sci. Chem., V. 41(1), p. 55-62, 1993.

[17] Emmi -S.S., D'Angelantonio -M., Beggiato -G., Camaioni -N.," The discrimination between triplet state and radical cation in the pulse radiolysis of bithiophene in $\mathrm{CCl}_{4}{ }^{n}, 9$. Tihany Symp. on Rad. Chem. Tata (Hungary) 29 Aug - 3 Sep $1998,[162$ p.] , p. O53, 1998.

[18] Cai-Zhongli ,"Study of Baicalin Scavenging Hydroxyethyl Peroxyl Radicals by Radiolysis of Aerated Ethanol-Baiclin System", Rad. Phys.and Chem. , V. $47(6)$, p. 896-871, 1996.

[19] Qian-Suping, Zhang-Jiashan, Yao-Side, Wang-Wenfeng, Lin-Nianyun," Scavenging of superoxide radical anion with hydroxy -cinnamic acid derivatives. A pulse radiolysis study", J. of Rad. Res. And Rad. Processing (Aug 1998), V. 16(3), p. 146-149, 1998.

[20] Truszkowski -S., Szymanski -W., "Stable Products and Radicals in the Radiolysis of Dichloromethane and 1,1,-Dichloroethane Gamma-Irradiated in an Oxygen-Free Atmosphere", J of Radioanal. and Nucl.Chem., V. 177(2) p. $415-423,1994$.

[21] Zubarev -V. E., Halpern -Alexander, "Spin Trapping of Radicals Formed Upon Irradiation of Organobromine Compounds with Low-Energy X-Ray", J. of the Chem. Soc. Faraday Trans., V. 90(5) , p. 721-726, 1994.

[22] Shimokawa -T., Nakagawa -S., "Genshiryoku-Kogyo, Development of Decomposition Method for Chloroflourocarbon (CFC) Solvent by Irradiation". V. $41(7)$, p. $15-19,1995$

[23] Rabani,-J., "Basic Aspects of Photocatalytic Detoxification of Organic Halogens by $\mathrm{TiO}_{2}$ Nanocrystallites. Photolytic and Radiolytic Investigations, 9. Tihany Symp.on Rad. Chem. ", Tata (Hungary) 29 Aug - 3 Sep 1998, p. 162,1998 
[24] Mincher -B. J., Arbon -R. E., Meikrantz -D. H., High Energy Decomposition of Halogenated Hydrocarbons., Idaho Falls , Final Report, United States Sep. 1992 , p. 15,1992

[25] Mao -Y., Schoeneich -C., Asmus -K.D.," Identification of Organic Acids and Other Intermediates of Chlorinated Ethanes on $\mathrm{TiO}_{2}$ Surfaces in Route to Mineralization. A Combined Photocatalytic and Radiation Study, Gamma Radiolysis", J. of Phys. Chem., V. 95(24) p. 10080-10089, 1991.

[26] Prager -L., Langguth -H., Rummel -S., Mehnert -R.," Electron Beam Degradation of Chlorinated Hydrocarbons in Air", Rad. Phys. and Chem., V. 46(4-6) , p. 1137-1142, 1995.

[27] Lian -Z.R., Yao -S.D., Lin -W.Z., Wang -W.F., Lin -N.Y.," Generation of $C_{60}$ Radical Cation and Radical Adduct of Dichloro- or Monochloromethyl Radical to $\mathrm{C}_{60}$ : Pulse Radiolysis and Laser Photolysis of $\mathrm{C}_{60}$ in Polar Chloromethane", Rad. Phys. and Chem., V. 50(3)., p. 245-247, 1997.

[28] Truszkowski -S., Szymanski -W., "Radiolysis of Chloropetane, 1 Chlorohexane and 1,6-Dichlorohexane", Nukleonica, V. 39(4), p. 21-34, 1994

[29] Shimokawa,-T. , Nakagawa -S. ,"Radiolysis of Halon", Tokyo-ToritsuAisotopu-Sogo-Kenkyusho-Nenpo (Sep 1997), p. 50-53, 1997.

[30] Szamrej -I., Tchorzewska -W., Kosc - H., Fory - M.," Thermal Electron Attahment Processes in Halomethanes. Pt. $1 . \mathrm{CH}_{2} \mathrm{Cl}_{2}, \mathrm{CHFCl}_{2}$ and $\mathrm{CF}_{2} \mathrm{Cl}_{2}{ }^{\prime \prime}$, Rad. Phys. and Chem., V. 47(2),p. 269-273, 1996.

[31] Kadhum,-A .A ., Salmon,-G . A ., "Electron Scavenging in the $y$-Radiolysis of tetrahydrofuran, Rad. Phys. and Chem. ", V. 23(1-2) p. 67-71, 1984.

[32] Zhang-Nan , Wu-Jilan," Using Multiple Scavenger Effects to Investigate the Radiolysis Mechanism of Cyclohexane and 4-Methyl-4-Phenyl-2-Pentanone System", J. of Rad. Res. and Rad. Processing (May 1992) , V. 10(2) , p. 109 112,1992

[33] Byakov, V.M., Nichiporov -F.G.," On the Mechanism of Formation of Radiolytic Hydrogen in Liquid Cyclohexane, Khimiya-Vysokikh-Ehnergij. (May-Jun 1996)", V. 30(3). p. 165-170., 1996.

[34] Podsoblyaev -A. P.," Radiation-Induced Oxidation of Cyclohexane: The Mechanism of Formation of Hydroperoxides", High Energy Chem. , V. 29(6) p. $440-442,1995$.

[35] Podsoblyaev -A. P.," Radiation Oxidation of Cyclohexane. Mechanism of Hydroperoxides Formation", High Energy Chem., V. 29(6), p. 407-409,
1995 
[36] Foeldiak -G., Roder -M. Wojnarovits -L., "Effect of LET on the Radiolysis of Cyclopentane", J. of Radioanal. and Nucl. Chem. V. 165(6), p. 385-394, 1992.

[37] Allayarov -S. R., Sablina -E. V., Barkalov -1. M., "Mehanism of Radiolysis of n-Perfluorohexane", High Energy Chem., V. 27(2), p. 100-105, 1993.

[38] Allayarov -S. R., Sablina -E. V., Barkalov -I. M.," On Radiolysis Mechanism of n-Perfluorohexane", High Energy Chem. , V. 27(2), p. 15-21, 1993.

[39] Drzewicz -P., Panta -P.; Gluszewski -W., Trojanowicz -M.," Effect of Selected Scavengers on Radiolytic Degradation of 2,4 -Dichlorophenol for Environmental Purposes", J. of. Radioanal. and Nucl. Chem., V. 242(3)., p. $601-609,1999$.

[40] Merga -G, Aravindakumar -C T , Rao -B. S. M. ," Pulse Radiolysis Study of the Reactions of $\mathrm{SO}_{4}^{-}$With Some Substituted Benzenes in Aqueous Solutions", J. of the Chem. Soc. Faraday Trans., V. 90(4) , p. 597-604, 1994.

[41]Bobrowski-K. ,Marciniak-B.,Hug-G.L. " 4-Carboxybezophenone -Sensitized Photooxidation of Sulfur-containing Amino Acids. Nanosecond Laser Flash Photolysis and Pulse Radiolysis Studies. Pulse Radiolysis", J. of the Amer. Chem. Soc. , V. 114, p. 10279-10288, 1992

[42] Jin - H., Pan - X., Wu-Jilan," Microsecond Pulse Radiolysis of Benzophenone Tributyl Phosphate System ", Rad. Phys. and Chem. , V. 47(6), p. 815-816, 1996.

[43] Choure -S.C., Bamatraf -M.M., Rao -B.S.M.," Radiation Chemical Oxidation of Cresols", TSRP-98: Trombay Symp.on Rad. and Photochemistry, Mumbai (India). 14-19 Jan 1998., p. 195-196, 1998.

[44] Kuruc -J., Sahoo -M. K.," GC-MS and GC-FTIR Identification of Volatile Radilytic Products in Water-Nitrobenzene-Carbon Tetrachloride Two Phase System", J. of Radioanal. and Nucl. Chem., V. 173(2) p. 395-407, 1993.

[45] Knighton -W. B., Sears -L. J.," Identification of Polychlorinated Biphenyl (PCB) Degradation Products in Gamma-Ray Irradiated Solutions of PCB's in Isopropanol", 41 American Soc. of Mass Spec. Conf. on Mass Spec. and Allied Topics, San Francisco, USA, 30 May - 4 Jun 1993.

[46] Patil -S. -F., Bedekar -A. G. Patil -R. M., " Gamma Radiolysis of Isobutanol and Nitrate in Aqueous Slution", J. of Radioanal. and Nucl. Chem. ..V. 158(1), p. 107-114, 1992

[47] Abramenkova -I. A., Batyuk -S. A., Bugaenko -L. T., Kalyazin -E. P.,"Mathematical Modelling of Methanol Radiolysis Kinetics. Communication 1. Gamma Radiolysis", Khimiya, V. 33(2), p. 141-146, 1992. 
[48] Abramenkova -I. A., Batyuk -S. A., Bugaenko -L. T., Kalyazin -E. P. Kovalev -G. V.," Mathematical Simulation Kinetics of the Methanol Radiolysis. 2. Mechanism of the Glycerin Formation", Gamma Radiolysis Khimiya, V. 33(3), p. 242-245, 1992.

[49] Ferradini -C., "Analysis of Liquid Methanol: A Review", Rad. Phys. and Chem., V. 48(4), p. $473-480,1993$.

[50] Jin-Haofang, Wu-Jilan, Fang-Xingwang, Zhang-Xujia," Selecting Ethanol as an Ideal Organic Solvent Probe In Radiation Chemistry Gamma-Radiolysis of Actone-Ethanol System and Acetophenone-Ethanol System", Japan Atomic Energy Inst.(JAERI) Conf., Mar 1995, p. 81-90, 1995.

[51] Torres, -L . , El-Desouky, -M ., Mathieu, -J ., Gorrichon, -J. P. ," Gamma Radiolysis of Furyl Ketones in 2-Propanol Solution", Rad. Phys. and Chem., V. $23(1-2)$, p. $121-126,1984$

[52] Maity -D. K., Mohan -H.," Electron Transfer reactions of 2,2 $2^{\prime}$-Thiodiethanoic Acid in Aqueous Slutions: A Pulse Radiolysis Study", 26 Inter. ESR Conf. Sheffield (United Kingdom), 29 Mar-2Apr. 1993.

[53] Belevskii -V. N., Belopushkin -S. I.," Mechanism of the Conversions of Intermediate Particles in Liquid-Phase Radiolysis of Acetic and Propionic Acids, Invetigated by ESR", High Energy Chem. , V. 25(5), p. 342-348, 1992.

[54] Bobrowski -K., Pogocki -D.," Mechanism of Acids Oxidation Contained Thioether Group by OH Radicals Generated by Radiation", 10 Nation. Con. Of Maria Skiodowska-Curie Polish Rad. Res. Soc. , Warsaw (Poland) , 6-7 Apr. 1995.

[55] Grigor ev -E. I., Nesterove -S. V.," Low Temperature Gamma-Radiolysis of Unsubstituted Crown Ehers", High Energy Chem. , V. 26(6) , p. 381-385, 1993.

[56] Faucitano -A., Buttafava -A., Patpuno -V.," The Radiation Chemistry of Perfluorinated Ethers" , Rad. Phys. and Chem. , V. 45(1), p. 23-30, 1995.

[57] Mincher -B.J., Arbon -R.E., Schwendimann -G.L., "Decomposition of PCBs in Oils Using Gamma Radiolysis", 96 Intern. Conf. on Nucl. and Hazardous Waste Management., Seattle, WA (United States). 18-23 Aug 1996., p. 252$257,1996$.

[58] Loele, -M. , Bazzanini, -R ., Chatilialoglu, -C . " Chemical Radiation Studies of 8-Bromoguanosine in Aqueous Solutions", J. Am. Chem. Soc., V. 122, p. $1900-1907$ 
[59] Mincher -B. J., Arbon -R. E., Radiolytic Removal of PCBs from Isooctane and Hydraulic Oil Solutions, Report by Lockheed Idaho Technologies Co., Idaho Falls, USA, 36 p. , 1996.

[60] Mincher -B.J., Arbon -R.E., Schwendimann -G.L., ," Decomposition of PCBs in Oils Using Gamma Radiolysis", Intern. Conf. on Riadioactive Waste Mang. And Environ. Remed. in Berlin 3-9 Sep. 1995, Fifth Intern. Conf. on Radioactive Waste Mang. And Environ. Remed. IC;M.95, Management. of Low Level waste and Remed. of Contamin. Sites and Facilities, New York 1995, American Soc. of Mech Eng. , (United States')., 1995.

[61] Kiryukhin -D.P., Kichigina -G.A., Mozhaev -P.S., Barkalov -I.M., "Crycopolimerization of Hydrogen Cyanide with Acetaldehyde", Vysokomolekulyarnye-Soedineniya-Seriya-A-i-Seriya-B, V 39(7). p. 11091114., 1997.

[62] Demidov -S.V., Kiryukhin -D.P., Mikhajlov,-A.I," On the Mechanism of LowTemperature Radiolysis of Vinyl Fluoride", Khimiya-Vysokikh-Ehnergij., V. 30(6). p. 418-420., 1996.

[63] Kudoh -H., Sasuga -T., Seguchi -T.," High Energy Ion Irradiation Effects on Polymer Materials.LET Dependance of $G$ Value of Scission of Polymethylmethacrylate PMMA", 7. intern. Symp. on Advanced Nucl. Energy Res. , Takasaki, Gunma (Japan). 18-20 Mar, p. 273-276., 1997.

[64] Yoshida-Hiroshi ,"Radiation-Degradation of Polymer Materials: Effect of Temperature, MC'96: Itern.Symp. on Material Chemistry in Nuclear Environment", Tsukuba, ibaraki (Japan)., p. 13-18, 14-15 Mar 1996

[65] Ichikawa -T., Oyama -K., Yoshida -H.," Mechanism of Radiation-Induced Degradation of Poly(Methy! Methacrylate)" 6 Japan-China Bilateral Symp. in Rad. Chem. , Tokyo (Japan), 6-11 Nov. 1994

[66] Szadkowska-Nicze -M., Mayer -J.," Pulse Radiolysis Studies of Poly(Ethylene Terephthalate) Film", 9. Tihany Symp.in Rad. Chem., Tata (Hungary), p. 162, 29 Aug - 3 Sep 1998.

[67] Celina -M., Gillen -K.T., Clough -R.L., International Atomic Energy Agency, Vienna (Austria), Final Report of Research Project, 1994-1997, p. 21-40, 1999

[68] Fouassier, -J . P., Rabek, -J . F., Radiation Curing in Polymer Science and Tec'nnology, Vol-1, Fundamentals and Methods, El Sevier Sci. Publ. Ltd. London, p. $351-353,1993$ 